\title{
Laparoscopic Retroperitoneal Para-aortic Lymph Node Biopsy in Advanced Cervical Cancer with Pelvic Lymph Node Metastases: A Single-centre Prospective Study
}

\author{
Kenta Yoshida \\ Mie University Hospital \\ Eiji Kondo ( $\square$ eijikon@clin.medic.mie-u.ac.jp ) \\ Mie University Hospital \\ Tsuyoshi Matsumoto \\ Mie University Hospital \\ Shintaro Maki \\ Mie University Hospital \\ Michiko Kaneda \\ Mie University Hospital \\ Masafumi Nii \\ Mie University Hospital \\ Toru Hirata \\ Mie University Hospital \\ Tomoaki Ikeda \\ Mie University Hospital
}

\section{Research Article}

Keywords: Cervical cancer, laparoscopy, retroperitoneal approach, para aortic lymph node

Posted Date: December 14th, 2020

DOI: https://doi.org/10.21203/rs.3.rs-123439/v1

License: @) (1) This work is licensed under a Creative Commons Attribution 4.0 International License. Read Full License

Version of Record: A version of this preprint was published at Journal of Obstetrics and Gynaecology Research on August 18th, 2021. See the published version at https://doi.org/10.1111/jog.14990. 


\section{Abstract}

Backgroud: Extended-field concurrent chemoradiation therapy (Ex-CCRT) is widely used for para-aortic lymph node (PAN) metastasis confirmed by radiographic assessment without surgical assessment. The objective of this prospective study was to evaluate the clinical value of laparoscopic retroperitoneal PAN biopsy in locally advanced cervical cancer (LACC) with pelvic lymph node metastases.

Methods: From May 2017 to March 2020, patients with stage IIB-IIIB cervical cancer, who were diagnosed with pelvic node metastasis using positron emission tomography-computed tomography (PET-CT) with SUVmax $\geq 2.0$, underwent laparoscopic retroperitoneal PAN biopsy. The radiation fields were extended to the PAN area with pathological metastases.

Results: Fourteen patients were diagnosed with squamous cell carcinoma of the cervix at FIGO stage IIB $(n=7)$ and IIIB $(n=7)$. The median operating time was 138 (range, 104-184) minutes. The median number of harvested PANs was 19 (range, 6-36). Three patients were diagnosed as positive for PAN metastasis by histological analysis. In this study, the sensitivity and specificity of PET-CT were $66.7 \%$ and $90.9 \%$, respectively.

Conclisions: The results of this study revealed that laparoscopic retroperitoneal PAN should be the standard treatment method worldwide because it is not appropriate to determine the radiation field of PAN by image examination without performing a histological assessment.

\section{Background}

In cervical cancer, metastasis to the para-aortic lymph nodes (PAN) is associated with a worse overall prognosis [1]. The rate of PAN metastasis in cervical cancer is $10-25 \%$. The rate of lymph node metastasis increases with the clinical stage; stage I (5\%), II (16\%), and III (25\%), respectively [2-4]. Until 2014, PAN metastasis in cervical cancer had stages up till IV B. In 2018, the clinical staging was revised by the International Federation of Gynecology and Obstetrics (FIGO) Committee to include a new clinical stage of cervical cancer: PAN metastasis without other distant metastases is classified as stage III C2 [5]. Aslan et al. reported positive pelvic lymph nodes (LNs) in 138/154 (74.6\%), positive pelvic and para-aortic LNs in 43/154 (23.3\%), and positive para-aortic LNs in only $4 / 154(2.2 \%)$ of the stage $\mathbb{C} 1$ and $\varangle \mathrm{C} 2$ cervical cancer patients [6]. Therefore, patients with positive pelvic LNs have a high risk of PAN metastasis, and determination of the PAN status is essential to ensure appropriate treatment planning for cervical cancer.

Concurrent chemoradiation therapy (CCRT) is the standard treatment for patients with locally advanced cervical cancer (LACC). The National Comprehensive Cancer Network guidelines recommend cisplatin-based chemotherapy plus extendedfield radiation therapy for patients with positive PANs [7]. The NCCN and the European Society for Medical Oncology (ESMO) guidelines recommend PAN biopsy before the administration of CCRT. To the best of our knowledge, the Gynecologic Oncology Group (GOG) trials showed a better prognosis of patients who underwent surgical staging than that of those who underwent radiographic staging [8].

However, the effectiveness of radiographic and surgical assessments is controversial. Most cases of para-aortic lymph node (PAN) metastasis are diagnosed by computed tomography (CT) or positron emission tomography (PET)-CT. Moreover, a metaanalysis reported that both magnetic resonance imaging (MRI) and CT have low sensitivities at $55.5 \%$ and $57.5 \%$, respectively [9]. Another meta-analysis reported that the pooled estimates for sensitivity and specificity of PET/CT were $0.71(95 \%$ confidence interval $[\mathrm{Cl}]=0.54-0.83)$ and $0.97(95 \% \mathrm{Cl}=0.93-0.98)$, respectively [10].

Since data on surgical staging is inadequate in Japan, this study aimed to evaluate the clinical value of laparoscopic retroperitoneal PAN biopsy in LACC with pelvic lymph node metastases diagnosed by PET-CT scan.

\section{Methods}


This prospective study was approved by the Mie University Medical Ethics Committee (registration number: No.3105, 12/5/2017) and registered with the University Hospital Medical Information Network (UMIN) (registration number:

UMIN000027458, 23/5/2017). This study was conducted following the Helsinki Declaration and Ethical Guidelines for Medical and Health Research Involving Human Subjects (MEXT, MHLW). All patients provided written informed consent.

\section{Inclusion criteria}

Women (age, 20-75 years) with FIGO stage II to III B (FIGO stage 2014) cervical cancer with histologically confirmed squamous cell carcinoma, adenosquamous carcinoma, or adenocarcinoma were included. All patients underwent magnetic resonance imaging (MRI) and PET-CT. Pelvic lymph nodes with SUVmax $\geq 2.0$ using PET-CT were considered positive.

\section{Exclusion criteria}

Potential subjects who met any of the following criteria were excluded from participating in this study: evidence of distant metastases other than PA lymph node metastases on imaging, unfit for laparoscopic surgery and CCRT, or WHO performance status over 2.

\section{Laparoscopic retroperitoneal para-aortic lymph node biopsy}

All surgical procedures were performed by the same surgical team. The patient was placed in the lithotomy position under anaesthesia. A 10-mm trocar for the introduction of the camera and intraperitoneal inspection was inserted sub-umbilically. A $10 \mathrm{~mm}$ trocar was inserted in the retroperitoneal space opposite McBurney's point. Carbon dioxide pneumoperitoneum was subsequently generated, keeping the intra-abdominal pressure $<10 \mathrm{~mm} \mathrm{Hg}$. Subsequently, two additional trocars, $10 \mathrm{~mm}$ and 5 $\mathrm{mm}$, were placed in the left abdomen. Following identification of the inferior mesenteric artery and the left ureter, a left PAN biopsy (the aorta up to the level of the left renal vein) was performed. The right PAN biopsy included the lymph nodes around the vena cava up to the right ovarian vein.

\section{Concurrent chemoradiation therapy}

All patients underwent planning CT in the supine position with 1-mm slices. Daily fractions of 1.8 Gy were given to the whole pelvic region 5 days a week to a total dose of 50.4 Gy. Brachytherapy was performed once a week with a fraction dose of 5-6 Gy; the total dose of the brachytherapy was 18-24 Gy in 3-4 fractions. If the PANs were histologically positive, extended CCRT (ex-CCRT) was performed. If needed, a boost to the parametrial region, pelvic lymph nodes, or PANs was administered with a single dose of $1.8 \mathrm{~Gy}$ up to $9 \mathrm{~Gy}$. All patients underwent brachytherapy based on MRI planning, and cisplatin $40 \mathrm{mg} / \mathrm{m}^{2}$ was administered once weekly during radiation for 5-6 consecutive weeks.

\section{Results}

The clinical characteristics of the study participants are shown in Table 1. The median follow-up period was 12 months (range, 3-28 months). All cases were diagnosed with FIGO stage II B $(n=7)$ and III $B(n=7)$ squamous cell carcinoma of the cervix with pelvic node metastases using PET-CT with SUVmax $\geq 2.0$. The median age was 55 years (range, 39-75 years), and the median BMI was $21.4 \mathrm{~kg} / \mathrm{m}^{2}$ (range, $16.4-30.9 \mathrm{~kg} / \mathrm{m}^{2}$ ). 
Table 1

clinical characteristics of study participants

\begin{tabular}{|ll|}
\hline $\mathbf{N}$ & 14 \\
\hline Median age, y (range) & $55(39-75)$ \\
\hline Median BMI, $\mathrm{kg} / \mathrm{m}^{2}$ (range) & $21.4(16.4-30.9)$ \\
\hline Histologic type & \\
\hline Squamous & 14 \\
\hline Tumor size, cm (range) & $5.6(3.9-6.7)$ \\
\hline SCC, ng/mL (range) & $12.0(1.3-104.5)$ \\
\hline Clinical stage & \\
\hline IIB & 7 \\
\hline IIIB & 7 \\
\hline
\end{tabular}

In this study, the median tumor size was $56 \mathrm{~mm}$ (range, 39-67 mm) at stage II B to stage III B. Before the initial therapy, SCC antigen had a high median level of $12.0 \mathrm{ng} / \mathrm{mL}$ (range, $1.3-104.5 \mathrm{ng} / \mathrm{mL}$ ). The median smallest diameter of the pelvic lymph node diagnosed using PET-CT with SUVmax $\geq 2.0$ was $14 \mathrm{~mm}$ (range, 6-26 mm).

Patient demographics, PET-CT results, and surgical outcomes are shown in Table 2. Three patients were diagnosed as positive for PA lymph node metastasis with PET-CT imaging. However, one of these patients was diagnosed as negative for PAN metastasis by histological analysis. The harvest lymph node numbers of that patient were 22 (Case 6 in Table 2). On the contrary, 11 patients were diagnosed as negative for PA lymph node metastasis with PET-CT imaging, and one of these patients was diagnosed as positive for PAN metastasis by histological analysis (Case 10 in Table 2). Thus, there was one false-positive case and one false-negative case with PET-CT (Fig. <link rid="fig1">1</link>-A,1-B), which had a sensitivity of $66.7 \%$ and a specificity of $90.9 \%$ in this study (Table 3 ). 
Table 2

Patient demographics and surgic outcomes

\begin{tabular}{|c|c|c|c|c|c|c|c|c|c|c|}
\hline Case & $\begin{array}{l}\text { Age } \\
\text { (v) }\end{array}$ & $\begin{array}{l}\text { BMI } \\
\left(\mathrm{kg} / \mathrm{m}^{2}\right)\end{array}$ & Stage & $\begin{array}{l}\text { SCC } \\
\text { (ng/mL) }\end{array}$ & $\begin{array}{l}\text { Tumor } \\
\text { size } \\
(\mathrm{mm})\end{array}$ & $\begin{array}{l}\text { Pelvic } \\
\text { lymph } \\
\text { node } \\
\text { size } \\
\text { by CT } \\
\text { scan } \\
(\mathrm{mm})\end{array}$ & $\begin{array}{l}\text { Pelvic } \\
\text { lymph } \\
\text { node } \\
\text { metastasis }\end{array}$ & $\begin{array}{l}\text { PAN } \\
\text { metastasis } \\
\text { (by PET- } \\
\text { CT) }\end{array}$ & $\begin{array}{l}\text { PAN } \\
\text { metastasis } \\
\text { (pathology*) }\end{array}$ & $\begin{array}{l}\text { Operation } \\
\text { time } \\
\text { (min) }\end{array}$ \\
\hline 1 & 39 & 21.4 & IIB & 10.7 & 67 & 15 & + & - & $0 / 22$ & 154 \\
\hline 2 & 46 & 20.5 & IIB & 14.9 & 44 & 12 & + & - & $0 / 36$ & 141 \\
\hline 3 & 44 & 27.1 & IIB & 52.5 & 42 & 16 & + & + & $7 / 14$ & 184 \\
\hline 4 & 48 & 16.7 & IIIB & 4.8 & 56 & 15 & + & - & $0 / 20$ & 104 \\
\hline 5 & 61 & 16.4 & IIIB & 12.0 & 53 & 24 & + & - & $0 / 19$ & 118 \\
\hline 6 & 67 & 29.3 & IIB & 3.4 & 60 & 8 & + & + & $0 / 22$ & 170 \\
\hline 7 & 55 & 29.3 & IIIB & 1.6 & 56 & 18 & + & - & $0 / 6$ & 134 \\
\hline 8 & 68 & 21.4 & IIIB & 104.5 & 56 & 15 & + & + & $5 / 10$ & 109 \\
\hline 9 & 69 & 21.4 & IIB & 3.6 & 55 & 6 & + & - & $0 / 14$ & 135 \\
\hline 10 & 55 & 18.9 & IIIB & 31.7 & 39 & 12 & + & - & $12 / 27$ & 128 \\
\hline 11 & 44 & 30.9 & IIIB & 1.3 & 40 & 13 & + & - & $0 / 14$ & 170 \\
\hline 12 & 40 & 22.3 & IIB & 6.1 & 65 & 26 & + & - & $0 / 31$ & 124 \\
\hline 13 & 58 & 27.6 & IIIB & 20.8 & 59 & 11 & + & - & $0 / 12$ & 153 \\
\hline 14 & 75 & 20.0 & IIB & 16.1 & 44 & 10 & + & - & $0 / 18$ & 142 \\
\hline
\end{tabular}

Table 3

Evaluation of PET-CT for detecting metastases to para-aortic lymph nodes ( $N=14)$

\begin{tabular}{|llll|}
\hline \multicolumn{3}{|c|}{ Pathological examination } \\
\hline & & PAN metastasis $(+)$ & PAN metastasis $(-)$ \\
\hline PET-CT & PAN metastasis (+) & 2 & 1 \\
\hline & PAN metastasis (-) & 1 & 12 \\
\hline Sensitivity, $66.7 \%$; Specificity, $92.3 \%$; Positive predictive value, $66.7 \%$; Negative predictive value, $92.3 \%$ \\
\hline False-positive rate, $7.7 \%$; False-negative rate, $33.3 \%$ \\
\hline
\end{tabular}

Furthermore, surgical outcomes were as follows: the median operating time was 138 (range, 104-184) minutes, and the median blood loss during the surgery was $3 \mathrm{ml}$ (range, 3-10). The median number of harvested PA lymph nodes was 19 (range, 6-36). The median time between surgery and CCRT administration was 13 (range, 11-17) days.

There were no complications during surgery in all the cases. After the operation, three patients developed lymphocysts which needed CT-guided drainage to plan the radiation field. One of them developed symptomatic lymphocysts. One case showed lung metastasis, identified in one case, was removed by thoracoscopy, and no further evidence of the disease was found. 


\section{Discussion}

In LACC, lymphatic spread typically occurs in a stepwise progression. The pattern of nodal metastasis tends to progress in a contiguous and predictable manner, starting from the lower pelvis to the upper pelvis, followed by the PANs. The regional pelvic lymph nodes become involved before the common iliac and para-aortic nodal chain and skip metastases are rare [11, 12]. In 2018, the clinical staging was revised by the FIGO Committee, and the most significant change in the existing staging system was the incorporation of nodal spread status as stage IIIC. Aslan et al. reported positive pelvic LNs in 138/154 (74.6\%), positive pelvic and para-aortic LNs in 43/154 (23.3\%), and positive para-aortic LNs only in 4/154 (2.2\%) of the stage $\mathbb{Z} C 1$ and $\triangle \mathrm{C} 2$ cervical cancer patients [6]. Patients with pelvic lymph node involvement are at a risk of occult PAN metastasis. Therefore, our prospective study only included patients with pelvic lymph node metastases diagnosed with PET-CT.

Radiographic and surgical examinations to diagnose lymph node metastasis have conflicting results. Radiographic assessment is useful, but it has low sensitivity. The criteria for lymph node involvement in CT and MRI are based on size and morphology. A node is suspicious when its shape is spherical, and the smallest diameter is greater than $10 \mathrm{~mm}$. Analysis of the outcome data from the GOG trials (GOG85, GOG120, and GOG165) showed a better prognosis for patients who underwent surgical exclusion of PAN involvement compared to those who underwent radiographic determination [8]. The results of a meta-analysis showed that both MRI and CT have low sensitivity (55.5\% and 57.5\%, respectively) [9]. In a meta-analysis conducted by Kang et al., the sensitivity of PET reached $73 \%$. However, in patients with negative morphological imaging, the sensitivity of PET-CT for the detection of microscopic lymph node metastases was much lower (almost 34\%) [13]. Another meta-analysis reported that the pooled estimates for sensitivity and specificity of PET-CT were 0.71 ( $95 \%$ confidence interval $[\mathrm{Cl}]=0.54-0.83)$ and $0.97(95 \% \mathrm{Cl}=0.93-0.98)$, respectively, [10]. In our study, the sensitivity and specificity of PET-CT were $66.7 \%$ and $90.9 \%$, respectively. In other words, $30 \%$ of the patients who underwent extended radiation for PAN metastasis diagnosed with PET-CT were over-treated. Further, $10 \%$ of the patients who did not undergo extended radiation for PAN negative diagnosed with PET-CT were under-treated.

Gouy et al. reported that the proportion of PANs showing no uptake on PET or PET-CT but were proven to be positive by histological analysis (false-negative rate) was $12 \%$, and the proportion of pelvic nodes with uptake on PET or PET-CT at the time of lymphadenectomy was $22 \%$ [2]. The present study also includes one false-negative case (Case 10, Fig. 1-B) and one false-positive case (Case 6, Fig. 1-A) with PET-CT. In this study, Case 10 had a high SCC antigen level (31.7 ng/mL) despite having the smallest tumor size (39 mm). The smallest size of pelvic lymph node metastases was $12 \mathrm{~mm}$, and the SUVmax was 5.2. In Case 6, the $S C C$ antigen level was low $(3.4 \mathrm{ng} / \mathrm{mL})$ despite the bulky tumor size $(60 \mathrm{~mm})$. The smallest size of pelvic lymph node metastases was $8 \mathrm{~mm}$, and the SUVmax was 3.5. Since this study is the small number of cases, the risk of false-negatives and false-positives was unknown. Large pelvic lymph node metastases (with the smallest diameter $18 \mathrm{~mm}$, $24 \mathrm{~mm}$, and $26 \mathrm{~mm}$ ) were not associated with the risk of PA lymph node metastases. The SCC antigen levels of three patients with PA lymph node metastases determined by pathological assessment were high $(31.7 \mathrm{ng} / \mathrm{mL}, 52.5 \mathrm{ng} / \mathrm{mL}, 104.5 \mathrm{ng} / \mathrm{mL})$. Our study hypothesizes that the SCC antigen level is related to PA lymph node metastases, but does not show this conclusively. Since the false-negative rate of radiographic assessment is high (33.3\% in this study), laparoscopic surgical PAN staging can identify the false-negative cases of PET-CT imaging.

In our study, three patients diagnosed with PA lymph node metastases by pathological staging were treated with Ex-CCRT. These PANs were low-volume disease $(\leq 5 \mathrm{~mm})$. Further, Gouy et al. reported the same survival rate for patients with PAN metastases $\leq 5 \mathrm{~mm}$ and patients without PAN involvement. In these cases, when imaging results are negative or inconclusive, surgery remains the best means to detect nodal disease definitively [3]. Therefore, if PA lymph node metastases with PET-CT were less than $5 \mathrm{~mm}$, surgical staging could be helpful as a prognostic factor. Moreover, the absence of false-positive cases could prevent unnecessary radiation therapy, as in Case 6 of the present study.

Our surgical procedure is a laparoscopic retroperitoneal approach, similar to previous studies [14]. The median number of lymph nodes retrieved was 19 (range, 6-36), which is more than that in the previous study. The median time in the initiation of radiotherapy was 13 days (range, 11-17 days). When there are no complications intraoperatively, CCRT can be started earlier

Page $6 / 10$ 
than laparotomy. Furthermore, the incidence of complications such as gastrointestinal ulcers, perforation, and ileus increases with an extended radiation field. The usefulness of IMRT has been reported earlier [15]. Although side effects could be reduced by IMRT in the future, laparoscopic retroperitoneal PAN with less intestinal obstruction than transperitoneal laparotomy should be the standard treatment worldwide.

In the present study, three patients (21.4\%) had lymphocysts that needed CT-guided drainage; one case was symptomatic. Lymphocysts are common (11-27\%), especially after retroperitoneal lymphadenectomy [16]. Our institution performs a similar surgery for endometrial cancer, and lymphocysts have occurred in only $2.5 \%(1 / 40)$ of the cases. The decrease in the occurrence of lymphocysts in these cases was due to minimum opening of the posterior peritoneum. CCRT had to be delayed in these three cases; therefore, we should be careful while making the incision larger.

Most cases of PA lymph node metastases are diagnosed using CT or PET-CT. In Japan, this is the first report of a surgical assessment using laparoscopic retroperitoneal approach, before administration of CCRT. The strength of our prospective study was the use of laparoscopic retroperitoneal para-aortic lymphadenectomy for LACC with pelvic lymph node metastases diagnosed with PET-CT, which is a more appropriate eligibility criterion than those used in previous retrospective reports. The identification of a false-positive case of PET-CT prevented the overtreatment of the patient. The limitation of our study was the small number of cases because it was a single-institution study. In the future, we will conduct a multi-institution study of LACC with pelvic lymph node metastases.

\section{Conclusions}

The results of this study revealed that laparoscopic retroperitoneal PAN should be the standard treatment method worldwide because it is not appropriate to determine the radiation field of PAN by image examination without performing a histological assessment.

\section{Abbreviations}

CCRT concurrent chemoradiation therapy

CT computed tomography

Ex-CCRT extended-field concurrent chemoradiation therapy

LACC locally advanced cervical cancer

MRI magnetic resonance imaging

PAN para-aortic lymph node

PET-CT positron emission tomography-computed tomography

\section{Declarations}

\section{Ethics approval and consent to participate}

This prospective study was approved by the Mie University Medical Ethics Committee (registration number: No.3105, 12/5/2017)

This study was conducted following the Helsinki Declaration and Ethical Guidelines for Medical and Health Research Involving Human Subjects (MEXT, MHLW).

All patients provided their written, informed consent. 


\section{Trial registration}

This prospective study was registered with the University Hospital Medical Information Network (UMIN) (registration number: UMIN000027458, 23/5/2017).

\section{Consent for publication}

All patients provided their written informed consent for the publication of the data.

\section{Availability of data and materials}

The raw data generated in this study are available from the corresponding author on reasonable request.

\section{Competing interest}

The authors declare no competing interests.

\section{Funding}

Not applicable.

\section{Authors' contribution}

K.Y., E.K., and T.I. developed the project, K.Y., E.K., T.H., and T.I. developed the protocols, E.K., T.M., S.M., and M.N. collected the data, K.Y. and M.K. analysed the data, K.Y. wrote the manuscript, E.K. edited the manuscript.

\section{Acknowledgements}

None

\section{References}

1. Stehman, F. B., Bundy, B. N., DiSaia, P. J., Keys, H. M., Larson, J. E., Fowler, W. C. Carcinoma of the cervix treated with radiation therapy: A multivariate analysis of prognostic variables in the gynecologic oncology group. Cancer. 67, 27762785 (1991).

2. Gouy, S., Morice, P., Narducci, F., Uzan, C., Gilmore, J., Leblanc, E. et al. Nodal-staging surgery for locally advanced cervical cancer in the era of PET. Lancet Oncol. 13, 212-220 (2012).

3. Gouy, S., Morice, P., Narducci, F., Uzan, C., Martinez, A., Leblanc, E. et al. Prospective multicenter study evaluating the survival of patients with locally advanced cervical cancer undergoing laparoscopic para-aortic lymphadenectomy before chemoradiotherapy in the era of positron emission tomography imaging. Clin. Oncol. 31, 3026-3033 (2013).

4. Vandeperre, A., Van Limbergen, E., Leunen, K., Moerman, P., Amant, F., Vergote, I. Para-aortic lymph node metastases in locally advanced cervical cancer: Comparison between surgical staging and imaging. Oncol. 138, 299-303 (2015).

5. Bhatla, N., Aoki, D., Sharma, D. N., Sankaranarayanan, R. Cancer of the cervix uteri. J. Gynecol. Obstet. 143, 22-36 (2018).

6. Aslan, K., Meydanli, M. M., Oz, M., Tohma, Y. A., Haberal, A., Ayhan, A. The prognostic value of lymph node ratio in stage IIIC cervical cancer patients triaged to primary treatment by radical hysterectomy with systematic pelvic and para-aortic lymphadenectomy. Gynecol. Oncol. 31, e1. doi: 10.3802/jgo.2020.31.e1. (2020). 
7. Cervical cancer. NCCN Clinical Practice Guidelines in Oncology (NCCN Guidelines), https://www.nccn.org/professionals/phisician_gls/pdf/cervical.pdf, Accessed date:20 August (2019).

8. Gold, M. A., Tian, C., Whitney, C. W., Rose, P. G., Lanciano, R. Surgical versus radiographic determination of para-aortic lymph node metastases before chemoradiation for locally advanced cervical carcinoma. Cancer Society. 13, 1954-1963 (2008).

9. Rockall, A. G., Sohaib, S. A., Harisinghani, M. G., Babar, S. A., Singh, N., Reznek, R.H. et al. Diagnostic performance of nanoparticle-enhanced magnetic resonance imaging in the diagnosis of lymph node metastases in patients with endometrial and cervical cancer. Clin. Oncol. 23, 2813-2821 (2005).

10. Yu, W., Kou, C., Bai, W., Yu, X., Duan, Ruixin., Yang, Y. et al. The diagnostic performance of PET/CT scans for the detection of para-aortic metastatic lymph nodes in patients with cervical cancer: A meta-analysis. PLoS One. 14, (2019).

11. Berman, M. L., Keys, H., Creasman, W., DiSaia, P., Bundy, B., Blessing, J. Survival and patterns of recurrence in cervical cancer metastatic to periaortic lymph nodes: a gynecologic oncology group study. Oncol. 19, 8-16 (1984).

12. Liu, Z., Hu, K., Liu, A., Shen, J., Hou, X., Zhang, F. et al. Patterns of lymph node metastasis in locally advanced cervical cancer. Medicine. 95, 4814 (2016).

13. Kang, S., Kim, S. K., Chung, D. C., Seo, S. S., Kim, J. Y., Park, S. Y. et al. Diagnostic value of 18F-FDG PET for evaluation of paraaortic nodal metastasis in patients with cervical cancer patients. Nucl. Med. 51, 360-367 (2010).

14. Ramirez, P. T., Jhingran, A., Macapinlac, H. A., Euscher, E. D., Munsell, M. F., Ramondetta, L. M. et al. Laparoscopic extraperitoneal para-aortic lymphadenectomy in locally advanced cervical cancer: A prospective correlation of surgical findings with positron emission tomography/computed tomography findings. Cancer. 117, 1928-1934 (2011).

15. Wu, Y., Zhu, B., Han, J., Xu, H., Gong, Z., Lu, E. et al. A comparative dosimetric study of cervical cancer patients with paraaortic lymph node metastasis treated with volumetric modulated arc therapy vs. 9-field intensity-modulated radiation therapy. Transl. Med. 7, (2019).

16. Moore, K. N., Gold, M. A., McMeekin, D. S., Walker, J. L., Rutledge, T., Zorn, K. K. Extraperitoneal para-aortic lymph node evaluation for cervical cancer via pfannenstiel incision; technique and peri-operative outcomes. Oncol. 108, 466-471 (2008).

\section{Figures}




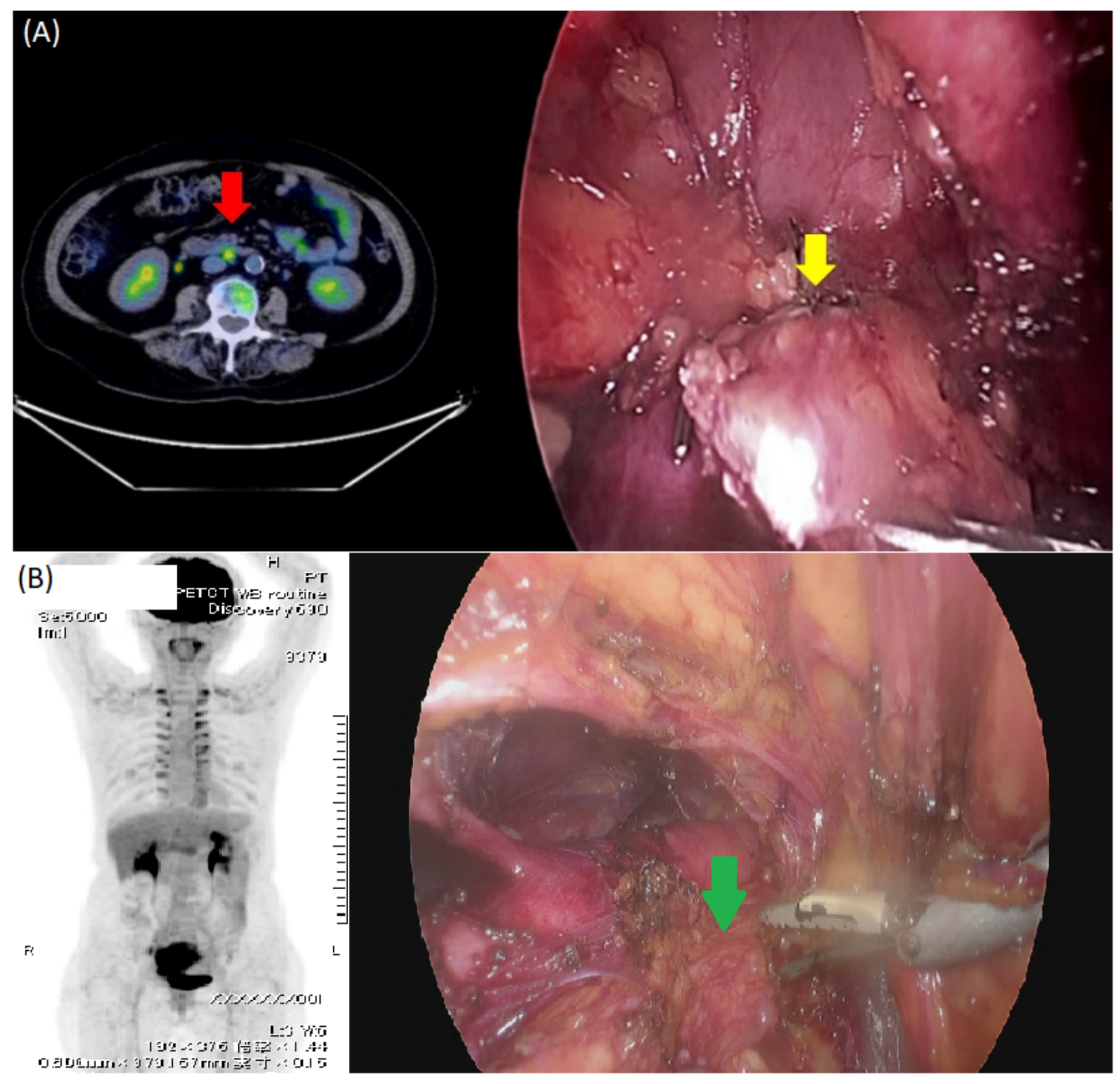

\section{Figure 1}

(A) Case 6 A false-positive case (Case 6) identified by laparoscopic retroperitoneal para-aortic lymph node (PAN) biopsy. Positron emission tomography-computed tomography (PET-CT) images of Case 6 show radiological metastasis (red arrow). PAN biopsy does not show any pathological metastasis (yellow arrow). (B) Case $10 \mathrm{~A}$ false-negative case (Case 10) identified by PAN biopsy. PET-CT images of Case 10 do not show any radiological metastasis. PAN biopsy revealed pathological metastasis (green arrow). 